\title{
Efficient Equilibrium Testing under Adhesion and Anisotropy using Empirical Contact Force Models
}

\author{
Kris Hauser \\ Dept. of Electrical and Computer Engineering \\ Duke University \\ Durham, North Carolina 27708 \\ Email: kris.hauser@duke.edu
}

\author{
Shiquan Wang and Mark Cutkosky \\ Dept. of Mechanical Engineering \\ Stanford University \\ Stanford, California 94305 \\ Email: \{shiquan,cutkosky\}@ stanford.edu
}

\begin{abstract}
A novel method is presented for efficiently testing the stability of an object under contact that accommodates empirically determined sets of admissible forces at contact points. These admissible force volumes may exhibit a wide variety of geometries, including anisotropy, adhesion, and even nonconvexity. The method discretizes the contact region into patches, performs a convex decomposition of a polyhedral approximation to each admissible force volume, and then formulates the problem as a mixed integer linear program. The model can also accommodate articulated robot hands with joint torques, joint frictions, and spring preloads. Predictions of our method are evaluated experimentally in object lifting tasks using a gripper that exploits microspines to exert strongly anisotropic forces.
\end{abstract}

\section{INTRODUCTION}

Contact force modeling is an essential component of grasp planning, physics simulation, end effector design, and biomechanics. The general purpose of such models is to simplify the countless microscopic interactions of a region of contact into a finite approximate representation that can be used for macroscopic predictions. Simple, mathematically convenient models like Coulomb friction are commonly used, but they fail to capture many phenomena including deformation, anisotropic friction, and adhesion, which are useful to exploit in engineering (e.g., novel surfaces [1, 11, 17] and micromanipulation [5]) as well as in understanding biological systems (e.g., the hairs on insect feet, Van der Waals forces on gecko feet, and claws on squirrels). For these complex interactions, an alternative approach is to use empirical data to capture the range of applicable forces during contact without slipping or separating [13, 14]. Although such data is useful for analysis and design, few methods use contact force data to make computationally efficient predictions.

This paper presents a computational method for testing static equilibrium of an object under empirical contact models. Empirical material-material contact modeling is conducted with a simple force measurement procedure that captures a force limit surface describing the maximum forces exhibited by a small contact unit in any given direction. Then, for an object touched by a novel assembly of contact units, its equilibrium status under an external wrench is predicted using a constrained optimization procedure. Unlike traditional force limit surfaces which bound conic and/or convex regions, this paper interested in supporting calculations with non-convex
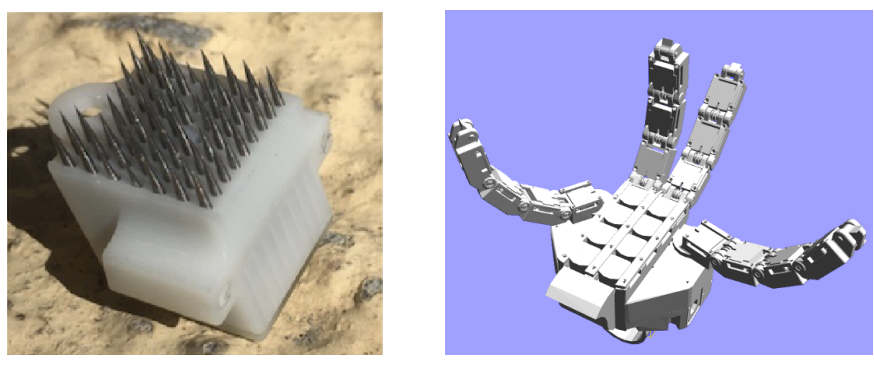

Fig. 1. The microspine unit used in this work (left) uses spring-loaded needles to yield high effective friction on rough surfaces. Our method uses empirical measurements of its applicable contact forces to predict the maximum loading characteristics of a hypothetical four-fingered gripper (right).

regions. To handle non-convex admissible force volumes, a mixed-integer linear programming (MILP) approach is presented that performs a branch-and-bound $(\mathrm{BnB})$ using a hierarchical convex decomposition. The method is globally optimal and significantly faster than off-the-shelf MILP solvers. The basic method is also extended to account for constraints in articulated robots, such as joint frictions, torques, and springs.

Experiments are conducted on a microspine unit that uses spring-loaded needles oriented at an angle to achieve high lateral loads on rocky surfaces [1, 21]. When pressed against a rough surface, several needles engage with asperities (indentations) on the surface leading to very large effective friction and slightly adhesive properties. We apply our method to design microspine grippers that handle objects with a given geometry and load. Our method is applied to microspine grasp analysis, in which the goal is to evaluate the lifting capability of a gripper at a given contact and finger configuration (Fig. 1). We compute wrench spaces for a variety of gripper configurations, and experiments show that even on problems of relatively modest size, the novel algorithm outperforms standard MILP solvers by one or more orders of magnitude.

\section{RELATED WORK}

The standard Coulomb friction point contact model is mathematically convenient, and allows for adequate and fast predictions about contact behavior between rigid objects such as force closure, optimal forces to resist an external wrench, static equilibrium, and dynamic simulation [2, 3, 4, 6, 18, 19]. 

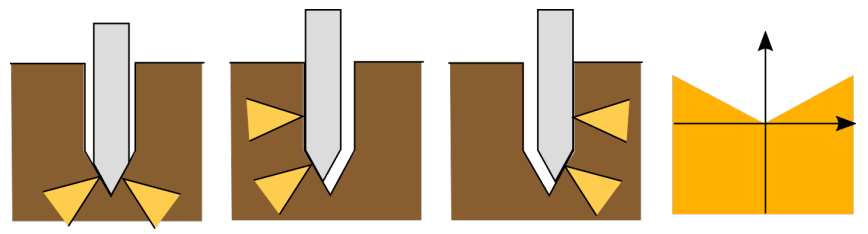

Fig. 2. Non-convex admissible force volumes (right) can arise out of microinteractions. Consider a peg-in-hole setup where the peg makes frictional contact with different sides of the hole depending on the external loading condition. Friction leads to apparent adhesion when shear loads are applied.

Mathematically, Coulomb friction may be expressed as a cone constraint, which leads to linear constraints in $2 \mathrm{D}$ or second-order cone constraints in 3D. A variety of conic extensions to the Coulomb model have been proposed, including a soft-finger approximation, polyhedral approximations, and anisotropic friction with elliptical constraints [2, 3].

Limit surfaces were introduced as a description of forces exerted on objects during planar sliding [9] and frictional contact [15] that are amenable to empirical testing. Similar empirical testing has been used to model soft finger contact [7] 20]. Novel devices that may be amenable to such modeling techniques include directional adhesive materials [12] and microspines [1]. More related to our work is Hawkes et al. [12], who use the limit surface of one adhesion unit to predict the feasibility of loading directions for two- and three-unit devices. However it does not handle non-convexity in the admissible force volume nor propose a computational method for general equilibrium prediction.

\section{Equilibrium with Empirical Force Models}

The general framework for our method is as follows:

1) Acquire limit surfaces defined locally with respect to a canonical surface-centric reference frame.

2) For a novel contact situation, estimate the contact region and split it into a finite number of contact patches. This yields a contact assembly.

3) Compute equilibrium prediction for the assembly under the estimated external wrench.

We will focus primarily on steps 1 and 3, and assume that the information in step 2 is provided through some other channels such as sensors or prior knowledge.

\section{A. Contact Model}

A contact region $C$ between bodies $O_{A}$ and $O_{B}$ is modeled as a rigid surface with a normal direction $n$ defined at each point $x \in C$. To handle anisotropy of friction forces, two orthogonal vector fields $u$ and $v$ are defined over $C$, which defines an orthogonal frame $R=(u, v, n)$ at all points $x \in$ $C$. The region is discretized into a finite number of contact patches $p_{1}, \ldots, p_{k}$. Each patch $i=1, \ldots, k$ is centered at the point $x_{i}$ and is associated with frame $R_{i}$.

At each contact patch, an admissible force volume $F_{i} \subseteq \mathbb{R}^{3}$ describes the set of valid forces $f_{i}$ applied to object $O_{B}$ at each point $x_{i}$. This volume is defined as the interior of the force limit surface $f_{\max }(d): S^{2} \rightarrow[0, \infty)$ which describes
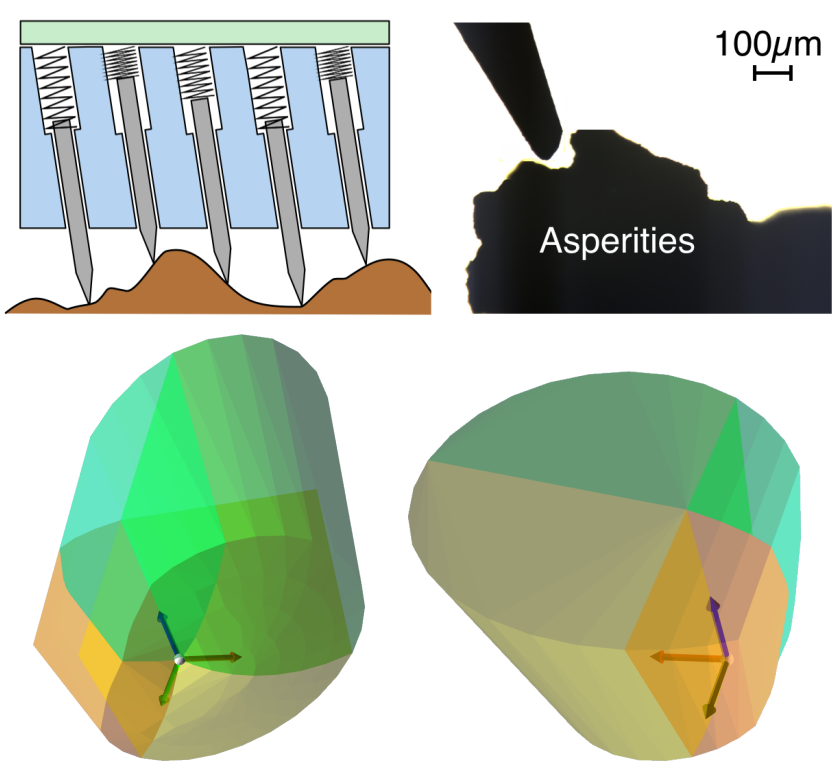

Fig. 3. Top: Diagram of a linearly-constrained microspine unit (left) and microscopic view of a spine about to catch on asperities (right). Bottom: two views of the admissible force volume for the microspine unit used in this work. Colors indicate different components of the volume's convex decomposition. Axes are labeled as follows: $x$ (red) the shear direction, $y$ (green) the lateral tangent direction, and $z$ (blue) the normal direction pointing into the unit. (Figure best viewed in color)

the maximum force in every direction in 3D. In other words $F_{i}=\left\{f \in \mathbb{R}^{3} \quad \mid \quad\|f\| \leq f_{\max }(\hat{f})\right\}$ where $\hat{f}=f /\|f\|$ is the unit vector in the direction of $f$. The world-oriented admissible force volume is a rotation of a local limit surface rotated by the frame $R_{i}$.

We measure a local limit surface described with respect to a canonical 3D reference frame aligned with $R=(u, v, n)$. For a given pair of materials, the local limit surface value in a given direction $d$ can be measured using a force sensor by applying a directional force to one object, keeping the other fixed, until the interface slips or otherwise breaks contact. For inward-pointing (compressive) directions, $f_{\max }(d)$ can either be infinite or capped by a maximum force that does not damage the object. For outward-pointing (separating) directions, $f_{\max }(d)$ will be 0 in the absence of adhesion. This process is repeated for a large number of directions. More details about this measurement procedure will be presented in Sec. IV.

Although the limit surface is a normally-displaced sphere, the admissible force volume may be non-convex, and we are particularly interested in handling these situations. Nonconvexity occurs in the case of surfaces that have multiple points of irregular micro-contact (Fig. 2), which can cause them to exhibit exotic adhesive behavior such as the microspine units presented below (Fig. 3). There may also be non-convex behavior in the compressive limits of buckling internal structures, such as corrugated cardboard which are stiffer in directions not parallel to the normal.

\section{B. Equilibrium Testing}

Equilibrium testing asks whether an external wrench $w_{\text {ext }} \equiv$ $\left(f_{\text {ext }}, t_{\text {ext }}\right) \in \mathbb{R}^{6}$ applied to $O_{B}$ can be resisted by forces at 
the contact points. Assume $t_{\text {ext }}$ is the external torque about the origin. Often, the external wrench is due only to gravity, the center of mass of $O_{B}$ is taken to be the origin, and hence $f_{\text {ext }}=m g$ and $t_{\text {ext }}=0$. Three conditions must be met for equilibrium to hold: force balance, torque balance, and admissibility of contact forces. In other words, it seeks an solution to the following feasibility problem:

Find $f_{1}, \ldots, f_{k}$ such that

$$
\begin{gathered}
\sum_{i=1}^{k} f_{i}+f_{\text {ext }}=0 \\
\sum_{i=1}^{k} x_{i} \times f_{i}+t_{\text {ext }}=0 \\
f_{i} \in F_{i} \text { for } i=1, \ldots, k
\end{gathered}
$$

For notational convenience, define the wrench matrix

$$
W=\left[\begin{array}{cccc}
I & I & \cdots & I \\
{\left[x_{1}\right]} & {\left[x_{2}\right]} & \cdots & {\left[x_{k}\right]}
\end{array}\right]
$$

where $[v]$ denotes the skew-symmetric cross product matrix, and define $\mathbf{f}=\left(f_{1}, \ldots, f_{k}\right)$ as the vector of contact forces. Problem (1) can then be expressed more compactly as

$$
\begin{gathered}
\text { Find } \mathbf{f} \text { such that } \\
W \mathbf{f}+w_{\text {ext }}=0 \\
f_{i} \in F_{i} \text { for } i=1, \ldots, k .
\end{gathered}
$$

To solve this problem efficiently and exactly when all of the $F_{i}$ are convex polyhedral regions, a linear program (LP) can be solved. This LP has $3 n$ variables and $6+n k$ constraints where $k$ is the number of faces bounding each $F_{i}$. However, alternative techniques are needed to solve the case of nonconvex regions.

\section{Joint torques}

To apply this method to an articulated robot, we enforce static equilibrium of forces and joint torques. This functionality is useful for determining whether equilibrium holds in the presence of passive joints or torque limits.

Let $O_{A}$ be the robot at configuration $q$ and $O_{B}$ be the object. Given the effects of gravity and a set of forces $f_{1}, \ldots, f_{k}$ that yield static equilibrium with $O_{B}$, the robot's joint torques $\tau$ must obey the following equilibrium balance equation:

$$
G(q)=\tau-\sum_{i=1}^{k} J_{i}(q)^{T} f_{i}
$$

where $G(q)$ is the generalized gravity vector and $J_{i}$ is the Jacobian of the $i$ 'th contact point. Note the introduction of the negative sign because the forces act on the object, while the equal and opposite force acts on the robot.

If sufficient torque were always available to the robot to enact the desired equilibrium balance, the force vector $\mathbf{f}$ could be solved independently from the torques, and torques calculated from (4). However, in the presence of torque limits, it may not be possible to enact such torques for a given solution $\mathbf{f}$, whereas contact force indeterminacy might allow for valid torques for some other solution $\mathbf{f}^{\prime}$.

As a result we incorporate torque limits $\tau_{\text {min }} \leq \tau \leq \tau_{\max }$ (inequalities taken element-wise) into constraints on $\mathbf{f}$ as follows:

$$
\tau_{\text {min }} \leq G(q)+\sum_{i=1}^{k} J_{i}(q)^{T} f_{i} \leq \tau_{\max }
$$

For fixed $q$ these inequalities are linear in $\mathbf{f}$.

\section{Wrench-space limit surface calculation}

A useful procedure for design of grasps and fixtures is to calculate the contact arrangement's wrench space $\mathcal{W} . \mathcal{W}$ is defined as the subset of wrenches $w_{\text {ext }} \in \mathbb{R}^{6}$ that can be resisted in equilibrium by admissible forces according to (3). We note that this is not necessarily a convex set. But it is apparent that for any point $w \in \mathcal{W}$, we can say that $c w \in \mathcal{W}$ for all $c \in[0,1]$. In other words, in order to determine $\mathcal{W}$ it suffices to calculate its limit surface $\partial \mathcal{W}$.

To do so we employ a method to optimize the external wrench in a given 6D direction such that equilibrium is maintained. Specifically, given some unit direction $\hat{w} \in \mathbb{R}^{6}$, a new variable $d$ is introduced into (3). Rather than only finding the contact force vector, $d$ is maximized subject to the equality constraint $w_{\text {ext }}=d \hat{w}$, i.e., we solve

$$
\begin{gathered}
\max _{\mathbf{f}, d} d \\
W \mathbf{f}+d \hat{w}=0 \\
f_{i} \in F_{i} \text { for } i=1, \ldots, k .
\end{gathered}
$$

Then, the limit surface can be obtained by sweeping $\hat{w}$ about the unit sphere in $6 \mathrm{D}, S^{5}$. In practice, to avoid requiring a excessive number of points, it can be more effective to approximate the wrench space by calculating its extents along different subspaces. Then $\mathcal{W}$ can be approximated as the intersection of the cylindrical extrusion of these extents back into $6 \mathrm{D}$ space.

\section{E. Separation Direction Prediction}

In the case that equilibrium does not hold, it may be valuable to predict which contact patches will separate and how the object will behave upon separation. Hence, we propose testing an alternative formulation that uses the maximum dissipation principle, which posits that frictional forces are determined to minimize the post-contact derivative of kinetic energy of the system. This condition seeks

$$
\begin{gathered}
\min _{\mathbf{f}} \frac{1}{2} \ddot{q}(\mathbf{f})^{T} B \ddot{q}(\mathbf{f}) \\
N W^{T} \ddot{q}(f) \geq 0 \\
f_{i} \in F_{i} \text { for } i=1, \ldots, k
\end{gathered}
$$

where $\ddot{q}(\mathbf{f}) \in \mathbb{R}^{6}$ is the post-forcing acceleration and angular acceleration (twist rate) of $O_{B}, B$ is the $6 \times 6$ mass matrix of the object, and $N$ is the $n \times 3 n$ block matrix of normal 
directions at each contact point $\operatorname{diag}\left(n_{1}^{T}, \ldots, n_{k}^{T}\right)$. We express $\ddot{q}(\mathbf{f})$ as

$$
\ddot{q}(\mathbf{f})=B^{-1}\left(W \mathbf{f}+w_{e x t}\right) .
$$

Hence, if all the $F_{i}$ are convex polytopes, then (7) can be solved exactly as a convex quadratic program.

\section{F. Branch-and-bound equilibrium solver}

We are now ready to present our primary contribution, which is a method for handling non-convex admissible force volumes in problem (3), with optional constraints (5). Given that these problems can be solved via a convex program when given convex admissible force volumes, we proceed by computing a convex decomposition of each of the $F_{i}$, and formulating a mixed integer linear program (MILP). First, we present the method in the simpler context of equilibrium testing (3), which asks for the first feasible solution.

Suppose each of the $k$ force volumes is decomposed into $c$ components, $F_{i}=F_{i, 1} \cup \cdots \cup F_{i, c}$ where each $F_{i, j}$ is a convex polyhedron. With each component given by a set of halfplanes $F_{i, j} \equiv\left\{f \in \mathbb{R}^{3} \quad \mid A_{i, j} f \leq b_{i, j}\right\}$, we formulate a MILP with ck indicator variables $z_{i, j} \in\{0,1\}$ as follows:

Find $\mathbf{f}, z_{1,1} \ldots z_{k, c}$ such that

$$
\begin{gathered}
W f+w_{\text {ext }}=0 \\
A_{i, j} f_{i} \leq b_{i, j}+M\left(1-z_{i, j}\right) \text { for } i=1, \ldots, k \text { and } j=1, \ldots, c \\
z_{i, j} \in\{0,1\} \text { for all } i, j \\
\sum_{j=1}^{c} z_{i, j}=1 \text { for all } i
\end{gathered}
$$

where $M$ is some large number (e.g., larger than the maximum radius of any force volume). Each of the indicators, when 1 , activates the constraints of the corresponding convex component. However, solving this MILP is usually prohibitively expensive because it is large (more than 10,000 constraints in many of our test problems) and may require exploring up to $c^{k}$ integer solutions, which is $7.9 \times 10^{28}$ in our largest problem with $c=4$ and $k=48$. As a result we use a BnB method, with a convex bounding volume hierarchy to speed up the search.

The general idea is to maintain for each $F_{i}$ a tree $T_{i}$ of convex volumes whose leaves are the components of the convex decomposition $F_{i, 1}, \ldots, F_{i, c}$. Each parent node stores the convex hull of all of its children, and hence the root node of $T_{i}$ stores $\operatorname{hull}\left(F_{i}\right)$. As a result, we can state the following facts about a given set $T_{1}, \ldots, T_{k}$ of trees or sub-trees. Let $S_{1}, \ldots, S_{k}$ be the root volumes of each of the given trees, and define $C P\left(S_{1}, \ldots, S_{k}\right)$ as the convex program (3) derived by replacing each $F_{i}$ with $S_{i}$. Then:

1) If $C P\left(S_{1}, \ldots, S_{k}\right)$ has no solution, there is no admissible set of contact forces in any combination of components amongst the leaves of $T_{1}, \ldots, T_{k}$.

2) If $C P\left(S_{1}, \ldots, S_{k}\right)$ has a solution $f_{1}, \ldots, f_{k}$ such that $f_{i} \in F_{i}$ for all $i=1, \ldots, k$, then it is a solution to (9).

3) If $C P\left(S_{1}, \ldots, S_{k}\right)$ has a solution $f_{1}, \ldots, f_{k}$ but some $f_{i} \notin F_{i}$ for some index $i$, then there may or may not be a solution amongst the leaves of $T_{1}, \ldots, T_{k}$.
The algorithm must branch only in Case 3. In order to minimize the amount of branching the algorithm is designed with three heuristics:

- The bounding hierarchy is constructed to minimize the deviation between a convex volume and the volume of $F_{i}$ contained therein.

- In Case 3, only a single tree $T_{i}$ selected for branching. The index $i$ and the order in which children of $T_{i}$ are tested are determined through heuristics described below.

- The objective function is constructed to prefer solutions close to the centroids of each of the $S_{i}$ 's.

a) Bounding hierarchy construction: To construct a convex hierarchy we use a bottom up approach. First, let the convex decomposition of a polyhedral set $A$ be given as $A_{1}, \ldots, A_{c}$. The convex hull of pairs of leaf volumes $B_{i}=$ $\operatorname{hull}\left(A_{2 i-1} \cup A_{2 i}\right)$ is constructed, and set as the parent of $A_{2 i-1}$ and $A_{2 i}$ in the hierarchy. If $c$ is odd, then we set $B_{\lceil c / 2\rceil}=A_{c}$. The process is repeated amongst the volumes $B_{1}, \ldots, B_{\lceil c / 2\rceil}$ until only one volume is reached, namely the convex hull of $A$. To minimize the amount of branching, it is important to choose the parent bounding volumes to be as close as possible to the union of the children. This increases the chance of finding an admissible solution at inner nodes of the tree (i.e., Case 2 above). Hence, we precompute the trees with a heuristic ordering of $A_{1}, \ldots, A_{c}$ as follows. For each pair of components $A_{i}$ and $A_{j}$ the hull $B_{i, j}=\operatorname{hull}\left(A_{i} \cup A_{j}\right)$ and its volume measure $\mu\left(B_{i, j}\right)$ are computed. The difference $\mu\left(B_{i, j}\right)-\mu\left(A_{i}\right)-\mu\left(A_{j}\right)$ is used as a priority score, with lower values having higher priority. Once a certain pair $\left(A_{i}, A_{j}\right)$ is chosen for merging, both components are removed from consideration and the next best pair is chosen, and so on. This process is repeated recursively for each stage in the hierarchy.

b) Branch ordering heuristics: When selecting a volume $T_{i}$ on which to branch, we pick the one that maximizes the distance to $F_{i}$ amongst all contact forces for which $f_{i} \notin F_{i}$. The children are then tested in order of increasing distance to the solution $f_{i}$.

c) Objective function heuristics: To maximize the likelihood of $C P\left(S_{1}, \ldots, S_{k}\right)$ finding a solution that is admissible (i.e., obeys Case 2 instead of Case 3), the objective function is designed to prefer forces respectively near the centroids of the union of the components in the leaves of $T_{1}, \ldots, T_{k}$. Specifically, for each $i=1, \ldots, k$ we find the union of components in the leaves of $T_{i}$, and compute its centroid $c_{i}$. Then, the objective function is defined as the L- $p$ norm

$$
g(\mathbf{f})=\sum_{i=1}^{k}\left\|f_{i}-c_{i}\right\|_{p}
$$

with $p$ either 1,2 , or $\infty$. In the case of $p=2$, the problem becomes a quadratic program which is a bit more computationally expensive than an LP, so we typically prefer $p=1$ or $p=\infty$.

\section{G. Branch-and-bound optimizer}

In optimization problems like (7) and calculating the wrench space of an assembly, the first valid solution may not be 
optimal. To optimize, we present an alternate $\mathrm{BnB}$ algorithm.

Again consider the convex program result on force volume subtrees $T_{1}, \ldots, T_{k}$ with root volumes $S_{1}, \ldots, S_{k}$. We maintain a known upper bound $\bar{g}$ to the optimal function value $g^{\star}$. We know that the optimal value $g$ to $C P\left(S_{1}, \ldots, S_{k}\right)$ is a lower bound on the optimal value of all combinations of leaves under $T_{1}, \ldots, T_{k}$. So, in each step of the method, there are three cases to consider.

1) If $C P\left(S_{1}, \ldots, S_{k}\right)$ has no solution, there is no admissible set of contact forces in any combination of components amongst the leaves of $T_{1}, \ldots, T_{k}$.

2) If $C P\left(S_{1}, \ldots, S_{k}\right)$ has a solution $f_{1}, \ldots, f_{k}$ with objective value $g$ such that $f_{i} \in F_{i}$ for all $i=1, \ldots, k$, then it at least as good as the solution in any combination of components amongst the leaves of $T_{1}, \ldots, T_{k}$. There is no need to branch.

3) If $C P\left(S_{1}, \ldots, S_{k}\right)$ has a solution $f_{1}, \ldots, f_{k}$ with objective value $g$ but some $f_{i} \notin F_{i}$ for some index $i$, then it may or may not contain an optimal solution.

In Case 2, if $g<\bar{g}$ then the optimal solution is set to $f_{1}, \ldots, f_{k}$ and the upper bound is set to $g$. In Case 3, if $g \geq \bar{g}$ then no sub-tree will contain a better solution, and hence search can be pruned at this point. However, if $g<\bar{g}$ then an optimal solution may or may not be contained in a sub-tree, which case requires branching. The following heuristics are used to speed up search.

a) Branch ordering heuristics: As above, we choose a single tree using the maximum distance heuristic. However, we use a priority queue maintained over all subtree sets, ordered by increasing objective function value $g$.

b) Early upper bound determination: When Case 3 occurs, we calculate the optimal forces at a fully-determined set of leaf nodes in the hope that it reduces the upper bound. To do so, for each subtree $T_{i}$ we pick a leaf such that if $f_{i} \in F_{i}$, the leaf is set to the component in which $f_{i}$ is contained, and if $f_{i} \notin F_{i}$, a random leaf is chosen.

c) $(1+\epsilon)$ relaxation: Instead of pruning on the bound $g<\bar{g}$, we relax this condition slightly to $g+\epsilon(1+|g|)<\bar{g}$, where $\epsilon$ is a small value (set to 0.001 in our experiments). This prunes out many more volumes in the presence of numerical errors and when redundancy in internal forces allows many optimal solutions amongst combinations of components. The resulting optimized value is guaranteed to be within $\epsilon\left(1+\left|g^{\star}\right|\right)$ of the true optimum.

\section{Microspine Experimental Setup}

A two-finger gripper (Fig. 5) based on linearly-constrained microspine units is built for the experiments. Microspines are hooks or needles with very sharp tips to catch and jam on micro structure of the surface, and are thus able to generate adhesion on rough surfaces (Fig. 3). The linear-constrained microspine unit design consists of straight needles that can slide along channels in the substrate, slightly pressed by the soft normal springs to maintain contact against the surface. The unit accommodates 60 linearly-constrained spines. This

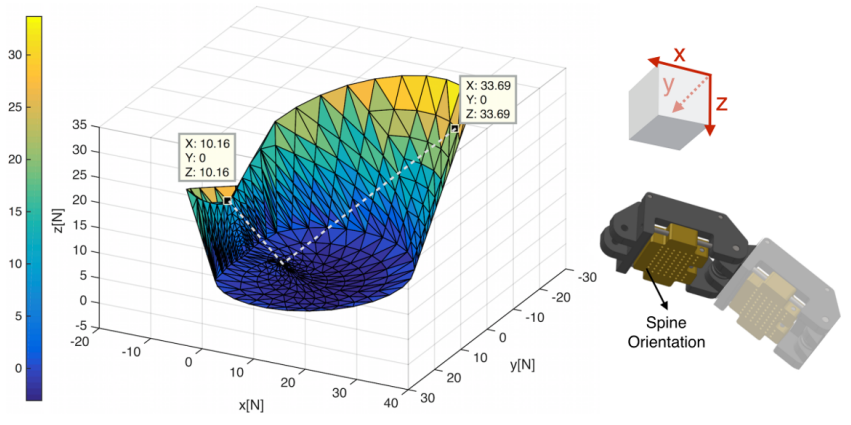

Fig. 4. Local limit surface (left): lumped contact property of the micro-spine unit on each gripper phalanx. The coordinate frame (upper right) is consistent with the assumed coordinate convention (lower right).

microspine unit is designed specifically for heavy-duty applications, featured with large adhesion density, low contact compliance and good comformality to surface variation [21]. Experiments are conducted to characterize the unit's contact properties and test the stability of passive grasps with the gripper under a variety of configurations and surface conditions.

a) Microspine unit limit surface acquisition: Contact friction of an individual spine engaged with an asperity can be described with the Coulomb model. However, when a group of spines interacts with terrain where the local curvature for each spine is randomly distributed, the overall contact property becomes complicated to model. It is inefficient to model many individual spines in contact because this requires detailed representation of the local contact geometry and kinematics of each spine. A more efficient method models 1D adhesion of a spine unit probabilistically based on empirical contact information of a single spine, which can then be extended to a 3D adhesion model that describes the adhesion profile over all loading directions (limit surface) of a spine unit.

The empirical data for a single spine contact includes maximum forces (150 data points per loading direction) that the spine can withstand before slipping, as measured by a force sensor. These data are described in more detail in [21]. The 3D limit surface (4) is derived based on how the adhesion degrades as the loading angle increases (away from the surface) due to decreasing number of usable asperities and increasing probability of non-spine-tip contact. With the assumption that all asperities are IID, the overall adhesion $F$ at any $2 \mathrm{D}$ loading angle ( $\mathrm{x}-\mathrm{z}$ plane) can be computed with the integral over all the usable asperity slopes $\psi$ :

$$
F(\phi)=\frac{\int_{\psi_{\min }(\phi)}^{\psi_{\max }(\phi)} C_{i}(\psi, \beta) C_{c}(\phi, \beta) d \psi}{\int_{\psi_{\min }}^{\psi_{\max }} C_{i}(\psi, \beta) C_{c}(0, \beta) d \psi} F(0)
$$

where $\phi$ is the loading angle from surface tangential (x axis), $\beta$ is inclination angle of the spine. $C_{i}$ and $C_{c}$ are decay factors due to non-spine-tip contact and spine slip along sliding

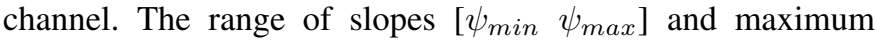
loading angle $\phi_{\max }$ can be estimated empirically. When considering $3 \mathrm{D}$ cases, the only parameter changes from $2 \mathrm{D}$ is 


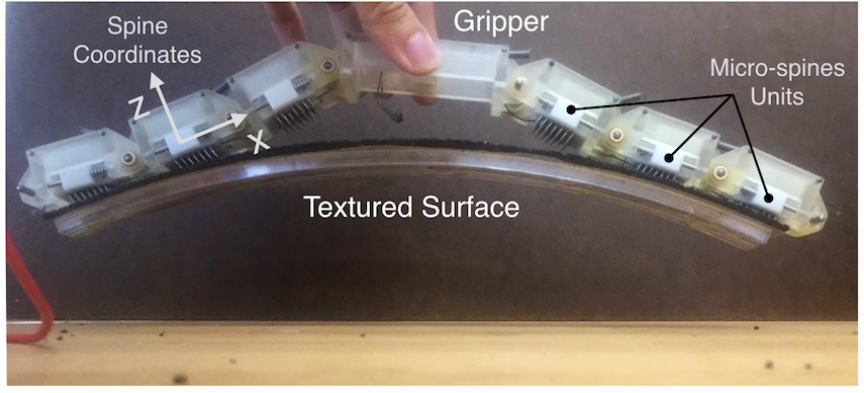

Fig. 5. Experiments equip a passive gripper with microspine units, which is lifted at the base to grasp a curved plate covered with roofing shingles. Different gripper configurations and surface curvatures are tested.

the equivalent spine inclination angle. With polar coordinate $(\phi, \theta)$ to denote the loading direction where $\theta$ represents the loading angle projected to the surface plane (x-y plane), the equivalent inclination angle $\beta^{\prime}$ becomes:

$$
\beta^{\prime}(\theta)=\arcsin (\sin \beta \cdot \cos \theta)
$$

which substitutes into equation (11) to compute the 3D limit surface for $z \leq 0$ (adhesion). Friction between spine substrate and contact surface is then included to model the other half of the limit surface. More details and empirical validation are available in a forthcoming paper [22].

The limit surface is shown in Fig. 4. Any possible force vector created by the spine unit should stay within this boatshaped hull. This non-convex surface shows that the spine unit adhesion degrades as the loading direction points more towards the surface normal (away from the contact surface) and eventually disappears. Due to the inclination of the spines, which are not perpendicular to the contact surface, there exists an optimal shear loading direction tangential to the surface, which is chosen as the $\mathrm{x}$ axis.

b) Passive gripper experimental device: A passive twofinger gripper is built with a spine unit per phalanx and three phalanges per finger (Fig. 57). The phalanges are passive, being connected with bearing-supported pin joints to reduce joint friction, with no springs or tendons to apply torque.

A set of laser-cut acrylic objects with different curvature are fabricated, then covered with roofing shingle to create a rough texture. In each test, the gripper is firstly pre-shaped and pushed against the object so several phalanges make contact. It is then lifted up by the base to grasp the surface passively: as the gripper moves upwards, the phalanges bend towards and "push" against the surface due to gravity, applying shear and adhesive forces (Fig. 5). Then, the gripper is slowly reoriented in various directions by hand until the object falls.

\section{NUMERICAL EXPERIMENTS}

We test our method with three assemblies: two springloaded opposed units (2OU), the passive two-finger gripper with 3 units per finger described above (2FG), and a candidate four-finger hand with actuation capabilities (4FH). The admissible contact forces for the 2FG are highly limited due
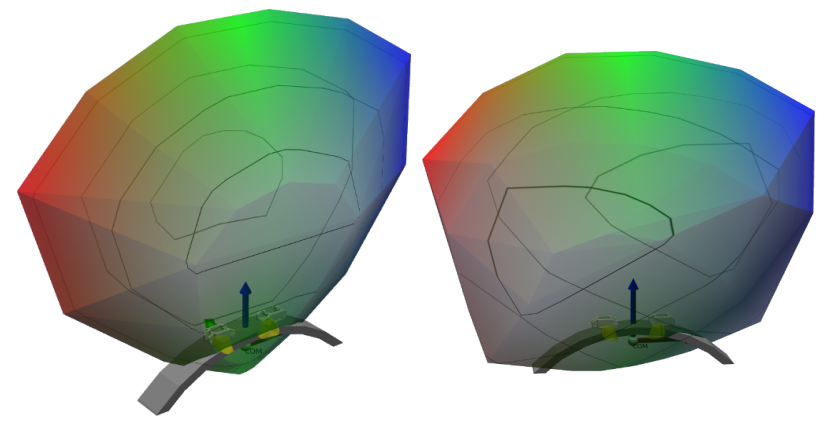

Fig. 6. 3D slices of the wrench space of the two-opposed unit (2OU) example lifting a curved object. Left: $\left(f_{x}, f_{y}, f_{z}\right)$ slice. Right: $\left(f_{x}, t_{y}, f_{z}\right)$ slice. Color indicates increasing $x$ coordinate. Level sets are indicated on the $y$ coordinate.

to low joint friction, which would cause buckling unless the net torque about the joint axis is low. As a result, torque constraints (5) are highly restrictive here. In contrast, 2OU and $4 \mathrm{FH}$ hand can exert active joint torques via springs, joint pulleys, and/or tendons. In these cases the primary limiting factors are shear friction and adhesion limits.

a) Contact and wrench space modeling: Each microspine unit that touches the object is considered "enabled." When enabled, the entire unit is assumed to make solid contact with the object, and is represented as a rectangular patch with four point contacts at the vertices. At each vertex, we define a force limit surface equal to $1 / 4$ the empirical force limit surface of the entire unit; this allows for a patch to resist torques, both parallel to and perpendicular to the contact normal. The admissible force volume is decomposed into 4 convex polyhedra, with $101,91,52$, and 52 vertices, respectively.

To illustrate the space of external wrenches we use the formulation of (6). These images are generated by sampling wrench directions from a 3D subspace of the wrench space using a regular sampling on a sphere. Below we use one of two 3D subspaces of the 6D wrench space: either (1) forces through the center of mass $\left(f_{x}, f_{y}, f_{z}\right)$, or (2) forces along the $\mathrm{X}-\mathrm{z}$ plane as well as torques about the normal $\left(f_{x}, t_{y}, f_{z}\right)$.

b) Opposed Units (2OU): The $2 \mathrm{OU}$ device combines two opposed units, spines pointing inward, connected via a springloaded slide. When contact is made between the units and a rough object, and the spring is loaded to apply an inward force, the spines engage the object. This allows for each unit to apply adhesive forces, and due to the admissibility of tangential shear forces, it can also apply stronger effective friction during compressive contact than would otherwise be possible.

Fig. 6 illustrates the limit surface of the assembly, which is is boat-shaped and elongated in the shear direction. Its $z$ coordinate also passes below 0 , which indicates the ability to resist downward pulling forces with adhesive contact forces. It can also resist large upward forces with large effective friction.

c) Passive Two-Finger Gripper ( $2 F G)$ : We compare the predictions of our method with the physical experiments of Fig 5. The masses of each link and the object were measured within $1 \mathrm{~g}$ accuracy, while centers of mass were estimated relatively coarsely. Joint friction is assumed to be $0.01 \mathrm{Nm}$. For each experiment we posed the simulated gripper posed 

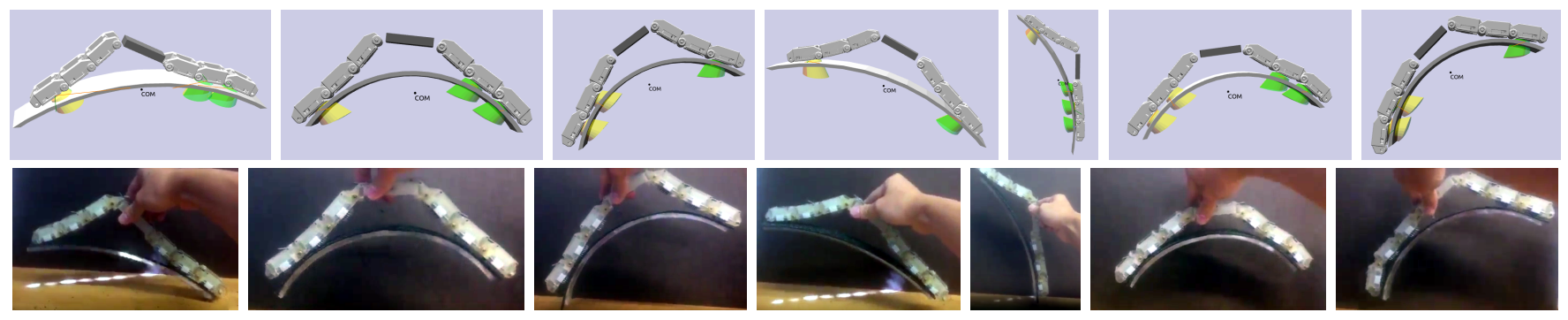

Fig. 7. Experiments exhibiting close agreement between predicted and actual onset of slip.
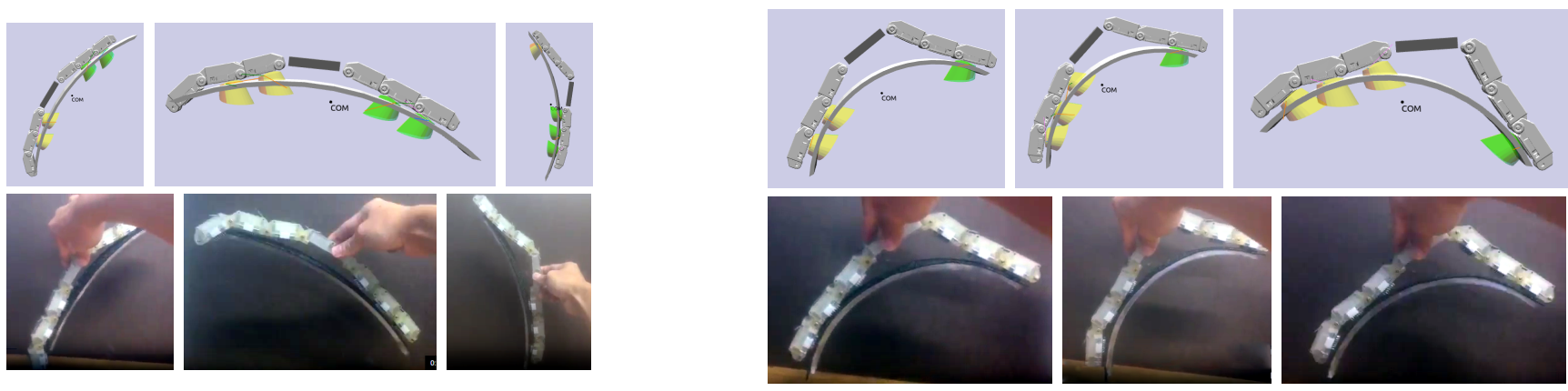

Fig. 8. Two experimental runs with inaccurate predictions. Left group: the model predicted the first slip event early (middle) and did not predict the final slip (right). Right group: the model did not predict the final slip (right).
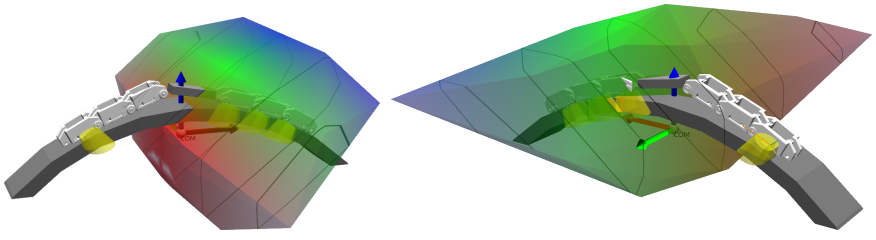

Fig. 9. 3D slices of the wrench space of the passive two-finger gripper (2FG) example in the distal-all phalanges (D-PMD) contact condition. Top: $\left(f_{x}, f_{y}, f_{z}\right)$ slice. Bottom: $\left(f_{x}, t_{y}, f_{z}\right)$ slice, rear view.

manually to match the physical gripper and observed which phalanges (P: proximal, M: medial, D: distal) made contact with the object. Configurations are denoted by the initials of the left and right phalanges in contact, separated by a dash, e.g., D-D, MD-MD, D-PMD, etc.

Fig. 7 shows that the method was able to accurately predict the angle of slip onset in 11/14 slip events. Fig. 8 shows the failure cases. In the first two, the left distal phalanx catches the tip of the object, and this contact is not included in our model. In the third, the left proximal link makes partial contact, and eventually separates. Our method fails to predict separation at this point because it (overconfidently) determines that the proximal and medial joints admit opposed forces that can produce net adhesive force. Excluding the proximal link leads to an accurate prediction of slip onset.

We also calculated wrench spaces for this device in different configurations. In general, the gripper can resist stronger loads in the $y$ direction because it is parallel to the passive joint axes and thus demands less joint friction to keep the links stationary. Also, the wrench space grows with more phalanges in contact. Fig. 9 and 10 illustrate two slices of the wrench spaces for the D-PMD and MD-MD configurations,

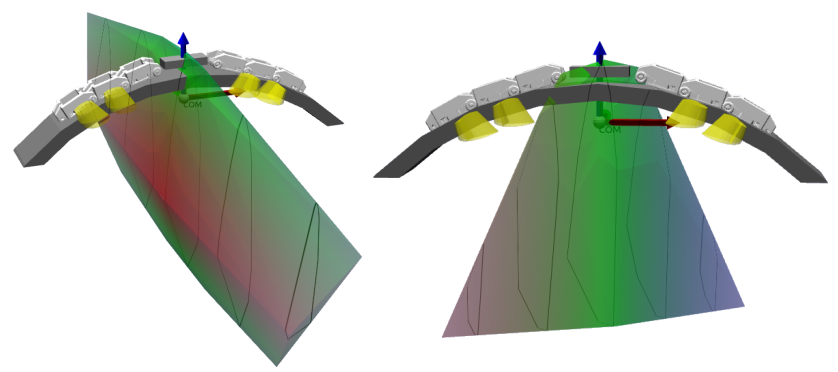

Fig. 10. 3D slices of the wrench space of the passive two-finger gripper ( $2 \mathrm{FG})$ example in the medial,distal-medial,distal (MD-MD) contact condition. Left: $\left(f_{x}, f_{y}, f_{z}\right)$ slice. Right: $\left(f_{x}, t_{y}, f_{z}\right)$ slice.
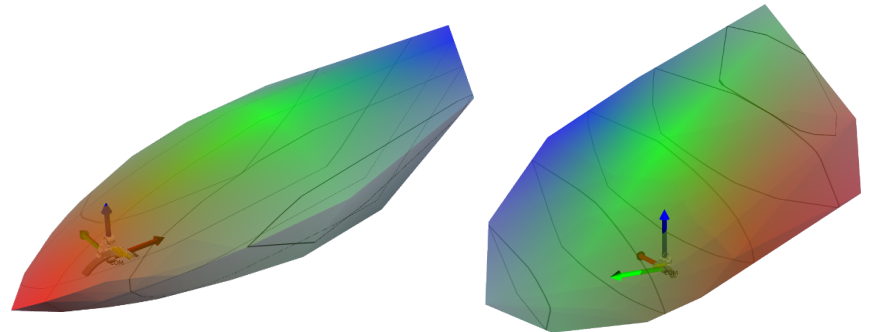

Fig. 11. 3D slices of the wrench space of the four-finger hand (4FH) example in the parallel (Par.) finger configuration. Top: $\left(f_{x}, f_{y}, f_{z}\right)$ slice. Bottom: $\left(f_{x}, t_{y}, f_{z}\right)$ slice, rear view.

respectively. The D-PMD configuration is able to support diagonal loads and stronger wrenches about the $y$ axis, since it can recruit 3 spine units to resist rightward shear. The MDMD configuration is able to support stronger downward forces because its symmetry allows shear forces to counterbalance one another. However, it is poor at resisting torques.

d) Four Finger Hand (4FH): The 4FH device is illustrated in Fig. 1. We investigate the differences in applicable wrenches when two outer fingers rotate between a parallel 
TABLE I

TIMING RESULTS

\begin{tabular}{|c|c|c|c|c|c|c|c|}
\hline Problem & Config. & \# units & \# CPs & Eq. (avg [min,max]) & Eq. SCIP (avg [min,max]) & Opt. (avg [min,max]) & \# CP $($ avg $[\min , \max ])$ \\
\hline $2 \mathrm{OU}$ & - & 2 & 8 & $25 \mathrm{~ms}[21 \mathrm{~ms}, 72 \mathrm{~ms}]$ & $254 \mathrm{~ms}[242 \mathrm{~ms}, 266 \mathrm{~ms}]$ & $38 \mathrm{~ms},[29 \mathrm{~ms}, 288 \mathrm{~ms}]$ & $1.1[1,11]$ \\
\hline $2 \mathrm{FG}$ & D-D & 2 & 8 & $55 \mathrm{~ms}[49 \mathrm{~ms}, 111 \mathrm{~ms}]$ & $527 \mathrm{~ms}[270 \mathrm{~ms}, 1.2 \mathrm{~s}]$ & $294 \mathrm{~ms}[29 \mathrm{~ms}, 6.7 \mathrm{~s}]$ & $10[1,271]$ \\
\hline $2 \mathrm{FG}$ & D-MD & 3 & 12 & $108 \mathrm{~ms}[51 \mathrm{~ms}, 336 \mathrm{~ms}]$ & $2.7 \mathrm{~s}[792 \mathrm{~ms}, 9.2 \mathrm{~s}]$ & $3.6 \mathrm{~s}[41 \mathrm{~ms}, 79.5 \mathrm{~s}]$ & $84[1,2246]$ \\
\hline $2 \mathrm{FG}$ & D-PMD & 4 & 16 & $366 \mathrm{~ms}[82 \mathrm{~ms}, 3.1 \mathrm{~s}]$ & $24 \mathrm{~s}[536 \mathrm{~ms}, 5 \mathrm{~m}+]$ & $8.3 \mathrm{~s}[57 \mathrm{~ms}, 178 \mathrm{~s}]$ & $154[1,3392]$ \\
\hline $2 \mathrm{FG}$ & MD-MD & 4 & 16 & $461 \mathrm{~ms}[73 \mathrm{~ms}, 23.8 \mathrm{~s}]$ & 50s $[492 \mathrm{~ms}, 5 \mathrm{~m}+]$ & $4.1 \mathrm{~s}[80 \mathrm{~ms}, 14.9 \mathrm{~s}]$ & $61[1,262]$ \\
\hline $2 \mathrm{FG}$ & MD-PMD & 5 & 20 & $180 \mathrm{~ms}[94 \mathrm{~ms}, 1.0 \mathrm{~s}]$ & 53s $[640 \mathrm{~ms}, 5 \mathrm{~m}+]$ & $10 \mathrm{~s}[87 \mathrm{~ms}, 267 \mathrm{~s}]$ & $159[1,4276]$ \\
\hline $2 \mathrm{FG}$ & PMD-PMD & 6 & 24 & $185 \mathrm{~ms}[136 \mathrm{~ms}, 288 \mathrm{~ms}]$ & $2.5 \mathrm{~s}[739 \mathrm{~ms}, 20 \mathrm{~s}]$ & $12 \mathrm{~s}[149 \mathrm{~ms}, 260 \mathrm{~s}]$ & $92[1,2272]$ \\
\hline $4 \mathrm{FH}$ & Par & 12 & 48 & $321 \mathrm{~ms}[298 \mathrm{~ms}, 396 \mathrm{~ms}]$ & $2.8 \mathrm{~s}[1.8 \mathrm{~s}, 15 \mathrm{~s}]$ & $2.6 \mathrm{~s}[226 \mathrm{~ms}, 28 \mathrm{~s}]$ & $10[1,271]$ \\
\hline $4 \mathrm{FH}$ & Opp & 12 & 48 & $581 \mathrm{~ms}[353 \mathrm{~ms}, 815 \mathrm{~ms}]$ & $1.8 \mathrm{~s}[1.6 \mathrm{~s}, 2.5 \mathrm{~s}]$ & $5.8 \mathrm{~s}[574 \mathrm{~ms}, 69 \mathrm{~s}]$ & $9.3[1,116]$ \\
\hline
\end{tabular}

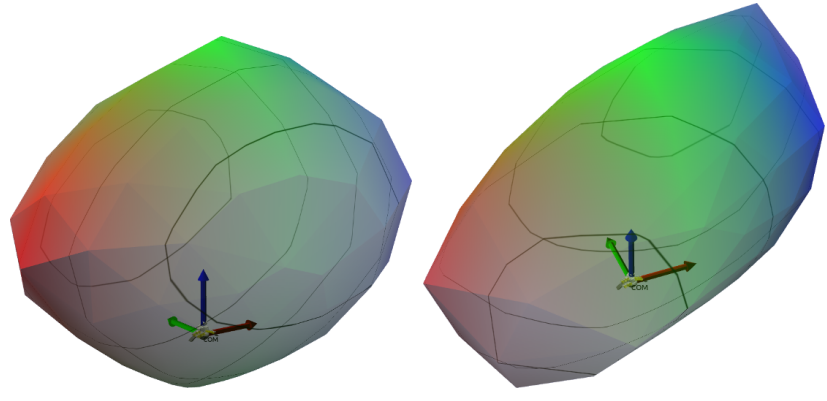

Fig. 12. 3D slices of the wrench space of the four-finger hand (4FH) example in the opposed (Opp.) finger configuration. Top: $\left(f_{x}, f_{y}, f_{z}\right)$ slice. Bottom: $\left(f_{x}, t_{y}, f_{z}\right)$ slice.

(Par.) and opposed (Opp.) configurations. Each joint is assumed to be able to exert a torque of $10 \mathrm{Nm}$. The fingers are slightly curved as through gripping a rock, and all 12 finger units are assumed to be engaged. Fig. 11 and Fig. 12 the wrench spaces for Par. and Opp., respectively. Par. can withstand heavy forces and torques in the finger-oriented direction because each unit is able to apply optimal shear. Opp. can better withstand downward loads because opposed shear forces are able to produce a net adhesion.

e) Implementation notes and running time: Our algorithm is implemented in the Python language, with CVXOPT as the convex program solver [16]. All experiments are performed on a single core of an 8 core Intel i7 processor. Sparse matrices are used for all linear inequalities. Some overhead may be reduced by implementation in a compiled language, but in our experiments over $80 \%$ of running time is spent inside the compiled convex program solver.

Experiments in Table I test equilibrium for 72 gravity directions in the range $\left[0^{\circ}, 360^{\circ}\right)$. Directional wrench optimization experiments are conducted for 135 directions in a sphere of forces through the object's center of mass. The leftmost columns give the problem, configuration, number of units, and number of contact points. Average, minimum, and maximum equilibrium testing time (Eq.), optimization time (Opt.), and number of convex program solves (\# CP) are given in the remaining columns. We observe a few notable trends:

- Average running time scales modestly in the number of contact points.

- Worst-case running time is 1-2 orders of magnitude worse than average.

- Worst-case scenarios happen rarely.
- Optimization is approximately an order of magnitude slower than feasibility testing.

The cases that exhibit worst-case behavior tend to be on the border between feasibility and infeasibility, which cause the solver to oscillate between combinations of integer solutions.

We compare against the MILP solvers in SCIP v3.2.1 [8] and Gurobi 7.0.2 [10]. Experiments show that SCIP is approximately 5-10x times slower than our algorithm when solutions were easily found. However, SCIP becomes extraordinarily slow in the worst case as the number of units grows. For example, in the D-PMD problem, for some directions of external load, SCIP was unable to find a solution after 1 hour of computation time. Hence, when generating the SCIP timing column (Eq. SCIP) all runs were capped at 5 minutes. In each row where the cap was reached (D-PMD, MD-MD, and MDPMD), $5-10 \%$ of runs failed. Gurobi performed with similar trends as SCIP, but was 1.5-4 times slower.

\section{CONCLUSION}

We presented a method for computing the quasi-static stability of an assembly of contacts under empirically-determined limit surfaces that supports anisotropy, adhesion, and nonconvexity. The mixed-integer linear programming (MILP) formulation is solved efficiently using a hierarchical convex decomposition and custom solver heuristics. Numerical tests demonstrate that it works orders of magnitude faster than an off-the-shelf MILP solver, and empirical tests suggest close agreement with experiments on a 2 fingered gripper.

Several promising avenues for future work remain. First, the solver is not yet fast enough for real-time simulation, and perhaps warm-starting would help in the presence of temporal consistency. Second, we only test the notion of weak equilibrium - verifying the existence of admissible forces in the best-case - whereas the notion of strong equilibrium may lead to more accurate predictions [18]. We also do not take into account hysteresis and deformation. Another important consideration is the stochastic nature of microscopic surface characteristics; properly handling this phenomenon would require new methods for computing the probability of equilibrium rather than a Boolean prediction.

\section{ACKNOWLEDGMENTS}

This work was supported by NSF NRI grant \#1527826. 


\section{REFERENCES}

[1] A. T. Asbeck, S. Kim, M. R. Cutkosky, W. R. Provancher, and M. Lanzetta. Scaling hard vertical surfaces with compliant microspine arrays. Int. J. Robotics Research, 25 (12):1165-1179, 2006. doi: 10.1177/0278364906072511. URL http://dx.doi.org/10.1177/0278364906072511.

[2] D. Baraff. Fast contact force computation for nonpenetrating rigid bodies. In Proc. 21st Annual Conf. Computer Graphics and Interactive Techniques, SIGGRAPH '94, pages 23-34, New York, NY, USA, 1994. ACM. ISBN 0-89791-667-0. doi: 10.1145/192161.192168. URL http://doi.acm.org/10.1145/192161.192168.

[3] A. Bicchi and V. Kumar. Robotic grasping and contact: a review. In IEEE Int. Conf. Robotics and Automation, volume 1, pages 348-353 vol.1, 2000. doi: 10.1109/ ROBOT.2000.844081.

[4] S. P. Boyd and B. Wegbreit. Fast computation of optimal contact forces. IEEE Trans. Robotics, 23(6):1117-1132, Dec 2007. ISSN 1552-3098. doi: 10.1109/TRO.2007. 910774.

[5] B. Brazey, R. Dahmouche, J.-A. Seon, and M. Gauthier. Experimental validation of in-hand planar orientation and translation in microscale. Intelligent Service Robotics, 9(2):101-112, Apr. 2016. ISSN 1861-2776. doi: 10.1007/s11370-015-0183-0. URL http://dx.doi.org/10. 1007/s11370-015-0183-0.

[6] T. Bretl and S. Lall. Testing static equilibrium for legged robots. IEEE Transactions on Robotics, 24(4):794-807, Aug 2008. ISSN 1552-3098. doi: 10.1109/TRO.2008. 2001360.

[7] A. Fakhari, M. Keshmiri, and I. Kao. Development of realistic pressure distribution and friction limit surface for soft-finger contact interface of robotic hands. $J$. Intelligent \& Robotic Systems, 82(1):39-50, 2016. ISSN 1573-0409. doi: 10.1007/s10846-015-0267-2. URL http://dx.doi.org/10.1007/s10846-015-0267-2.

[8] G. Gamrath, T. Fischer, T. Gally, A. M. Gleixner, G. Hendel, T. Koch, S. J. Maher, M. Miltenberger, B. Müller, M. E. Pfetsch, C. Puchert, D. Rehfeldt, S. Schenker, R. Schwarz, F. Serrano, Y. Shinano, S. Vigerske, D. Weninger, M. Winkler, J. T. Witt, and J. Witzig. The scip optimization suite 3.2. Technical Report 15-60, ZIB, Takustr.7, 14195 Berlin, 2016.

[9] S. Goyal, A. Ruina, and J. Papadopoulos. Limit surface and moment function descriptions of planar sliding. In International Conference on Robotics and Automation, pages 794-799 vol.2, May 1989. doi: 10.1109/ROBOT. 1989.100081.

[10] Gurobi Optimization, Inc. Gurobi optimizer version 7.0.2, 2017. URL http://www.gurobi.com

[11] E. W. Hawkes, D. L. Christensen, E. V. Eason, M. A. Estrada, M. Heverly, E. Hilgemann, H. Jiang, M. T. Pope, A. Parness, and M. R. Cutkosky. Dynamic surface grasping with directional adhesion. In IEEE/RSJ Int. Conf. Intelligent Robots and Systems, pages 5487-5493,
Nov 2013. doi: 10.1109/IROS.2013.6697151.

[12] E. W. Hawkes, H. Jiang, and M. R. Cutkosky. Threedimensional dynamic surface grasping with dry adhesion. Int. J. Robotics Research, 35(8):943-958, July 2016. ISSN 0278-3649. doi: 10.1177/0278364915584645. URL http://dx.doi.org/10.1177/0278364915584645.

[13] R. D. Howe and M. R. Cutkosky. Practical forcemotion models for sliding manipulation. Int. J. Robotics Research, 15(6):557-572, 1996. doi: 10.1177/ 027836499601500603. URL http://dx.doi.org/10.1177/ 027836499601500603

[14] R. Kolbert, N. C. Dafle, and A. Rodriguez. Experimental validation of contact dynamics for in-hand manipulation. In Int. Symp. Experimental Robotics, 2016.

[15] S. H. Lee and M. R. Cutkosky. Fixture planning with friction. J. Engineering for Industry, 113(3):320-327, Aug. 1991. doi: 10.1115/1.2899703. URL http://dx.doi. org/10.1115/1.2899703.

[16] J. D. M. S. Andersen and L. Vandenberghe. Cvxopt: A python package for convex optimization, version 1.1.5, 2012. URL http://abel.ee.ucla.edu/cvxopt

[17] C. Majidi, R. Groff, Y. Maeno, B. Schubert, S. Baek, B. Bush, R. Maboudian, N. Gravish, M. Wilkinson, K. Autumn, and R. Fearing. High friction from a stiff polymer using micro-fiber arrays. Physical Review Letters, 97(076103), Aug. 2006.

[18] J. S. Pang and J. C. Trinkle. Stability characterizations of fixtured rigid bodies with coulomb friction. In IEEE Int. Conf. Robotics and Automation, volume 1, pages 361368 vol.1, 2000. doi: 10.1109/ROBOT.2000.844083.

[19] D. E. Stewart. Rigid-body dynamics with friction and impact. SIAM Review, 42(1):3-39, 2000. doi: 10.1137/S0036144599360110. URL http://dx.doi.org/10. 1137/S0036144599360110.

[20] P. Tiezzi and I. Kao. Modeling of viscoelastic contacts and evolution of limit surface for robotic contact interface. IEEE Trans. Robotics, 23(2):206-217, April 2007. ISSN 1552-3098. doi: 10.1109/TRO.2006.889494.

[21] S. Wang, H. Jiang, and M. R. Cutkosky. A palm for a rock climbing robot based on dense arrays of microspines. In IEEE/RSJ Int. Conf. Robots and Systems, pages 52-59. IEEE, 2016.

[22] S. Wang, H. Jiang, and M. Cutkosky. Design and modeling of linearly-constrained compliant spines for humanscale locomotion on rocky surfaces. Int. J. Robotics Research (to appear), 2017. 